\title{
Cyclosporine A prevents apoptosis-related mitochondrial dysfunction after neonatal cardioplegic arrest
}

Norihiko Oka, MD, Lixing Wang, MD, PhD, Wenyu Mi, MD, Wei Zhu, MD, Osami Honjo, MD, and Christopher A. Caldarone, MD

Supplemental material is available online.
Objective: Mitochondrial permeability transition pore opening plays a critical role in mediating the mitochondrial response to ischemia/reperfusion injury and initiation of apoptosis. We tested whether inhibition of mitochondrial permeability transition pore opening with cyclosporine A prevented apoptosis-related alterations in mitochondrial structure and function after cardioplegic arrest.

Methods: Newborn piglets (age $\sim 14$ days) underwent cardiopulmonary bypass, cardioplegic arrest (60 minutes), weaning from bypass, and 6-hour reperfusion. Comparison was made among cold crystalloid cardioplegia $(n=5)$, cold crystalloid cardioplegia with cyclosporine A pretreatment $(n=5)$, and noncardiopulmonary bypass $(\mathrm{n}=5)$ groups.

Results: Early apoptosis signaling events (Bax translocation to the mitochondria) were prominent in cold crystalloid cardioplegia and prevented in cold crystalloid cardioplegia + cyclosporine A myocardium. Mitochondrial release of cytochrome c, determined by Western blot of cytosolic fractions and confocal quantitative colocalization analysis, was also prominent in cold crystalloid cardioplegia but prevented in cold crystalloid cardioplegia + cyclosporine A myocardium. Electron microscopy of isolated mitochondria demonstrated subjective alterations in mitochondrial architecture in cold crystalloid cardioplegia mitochondria, which were prevented by cyclosporine A. Deficiency of isolated mitochondrial oxygen consumption at Complex I was present in cold crystalloid cardioplegia mitochondria and prevented by cyclosporine A $(P<.01)$. The frequency of deoxyuride-5'triphosphate biotin nick end labeling-positive myocytes was diminished in cold crystalloid cardioplegia + cyclosporine A myocardium $(P<.05)$. Mitochondrial resistance to calcium-mediated mitochondrial permeability transition pore opening was not different in cold crystalloid cardioplegia and noncardiopulmonary bypass mitochondria, suggesting that calcium overload is not solely responsible for the observed deficits in mitochondrial function.

Conclusions: Cyclosporine A pretreatment prevents postcardioplegia alterations in mitochondrial structure and function in a clinically relevant model of neonatal cardiac surgery. Prevention of mitochondrial permeability transition pore opening and apoptosis signaling events (Bax translocation and mitochondrial permeabilization) are associated with superior mitochondrial preservation.

$\mathrm{T}$ The early postoperative period after neonatal cardiac surgery is notable for myocardial dysfunction. ${ }^{1,2}$ Recent data indicate that apoptosis is induced after cardioplegic arrest, suggesting a potential role for apoptosis in postoperative myocardial dysfunction. ${ }^{3,4}$ As a critical mediator in apoptosis signaling pathways, mitochondria integrate upstream death stimuli and undergo structural and functional remodeling with subsequent transmission of signals to downstream executioner proteins. ${ }^{5}$ We previously identified Bax translocation to the mitochon- 


$$
\begin{aligned}
& \text { Abbreviations and Acronyms } \\
& \mathrm{CCP}=\text { cold crystalloid cardioplegia } \\
& \text { COX IV }=\text { cytochrome } \mathrm{c} \text { oxidase IV } \\
& \mathrm{CPB}=\text { cardiopulmonary bypass } \\
& \text { CsA }=\text { cyclosporine } \mathrm{A} \\
& \text { Ig = immunoglobulin } \\
& \text { IV } \quad=\text { intravenous } \\
& \text { MPTP }=\text { mitochondrial permeability transition pore } \\
& \text { OCT = optimal cutting temperature } \\
& \text { TUNEL }=\text { deoxyuride-5' } \text {-triphosphate biotin nick end }
\end{aligned}
$$

dria, mitochondrial permeabilization, cytochrome c release, and deficits in mitochondrial Complex I function within 6 hours after cardioplegic arrest and reperfusion. ${ }^{6,7}$ Because these events occur in a time frame similar to that of postoperative cardiac dysfunction, we hypothesized that apoptosis-related mitochondrial dysfunction may contribute to postoperative myocardial dysfunction. ${ }^{6}$

The mitochondrial permeability transition pore (MPTP) is a nonspecific pore that has been implicated as a potential mediator of myocardial ischemic injury and initiation of apoptosis. ${ }^{8,9}$ Pharmacologic inhibition of MPTP opening is associated with myocardial protection against ischemic/ reperfusion injury. ${ }^{10,11}$ Cyclosporine A (CsA), which acts by inhibiting the peptidyl-prolyl cis-trans isomerase activity of CyP-D, is a specific inhibitor of MPTP opening, and the protective effects of CsA after ischemic injury have been reported in a variety of in vitro ${ }^{12,13}$ and in vivo ${ }^{14}$ animal models of unprotected and protected ${ }^{15}$ ischemia. The potential for CsA-mediated MPTP inhibition to mitigate mitochondrial injury after cardioplegic arrest has rarely been explored in a large animal in vivo model. Therefore, the current study was designed to examine the effect of CsA on mitochondrial injury and apoptosis signaling in a clinically relevant model of neonatal cardioplegic arrest.

\section{Materials and Methods \\ Surgical Preparation}

Newborn piglets $(\sim 14$ days) were anesthetized with intravenous (IV) sodium pentothal, intubated, and maintained with $1 \%$ isoflurane. Cardiopulmonary bypass (CPB) was initiated with a blood prime and passive cooling to $28^{\circ} \mathrm{C}$ to $30^{\circ} \mathrm{C}$ core temperature. The aorta was crossclamped, and cold antegrade crystalloid cardioplegia (Plegisol; Hospira, Inc, Lake Forest, Ill) $\left(4^{\circ} \mathrm{C}, 50-70 \mathrm{~mm} \mathrm{Hg}\right.$ perfusion pressure; $20 \mathrm{~mL} / \mathrm{kg}$ followed by $15 \mathrm{~mL} / \mathrm{kg}$ every 20 minutes) was administered. Topical cooling and aorta and pulmonary artery venting were used. After 60 minutes, the crossclamp was removed and the animal was rewarmed; after 10 to 20 minutes, CPB was terminated. General anesthesia was maintained for 6 hours. Arterial blood gases, electrolytes, and hematocrit were maintained in a physiologic range. Inotropic agents were not administered.
After 6 hours, the heart was quickly excised and perfused with ice-cold phosphate-buffered saline. Left ventricular myocardium was excised, snap-frozen in liquid nitrogen, and stored $\left(-80^{\circ} \mathrm{C}\right)$. For mitochondrial oxygen consumption and cytochrome $\mathrm{c}$ release measurements, myocardium was immediately fractionated as described. ${ }^{6}$ Fresh mitochondrial fractions were used for oxygen consumption measurements, mitochondrial calcium tolerance experiments, and electron microcopy. Myocardium was embedded in optimal cutting temperature (OCT) compound, frozen in liquid nitrogen, and stored at $-80^{\circ} \mathrm{C}$ for apoptotic index assessment and fluorescent immunohistochemistry. Mitochondrial purity was confirmed by inspection of randomly chosen electron micrographs and by porin concentrations and citrate synthase activity.

Comparison was made among the following groups: cold crystalloid cardioplegia (CCP, $\mathrm{n}=5$ ); $\mathrm{CCP}$ with IV administration of CsA (10 mg/ $\mathrm{kg}^{-1}$; Novartis Pharmaceuticals Canada Inc, Dorval, Quebec $)^{14}$ immediately before initiation of CPB (CCP + CsA, $\mathrm{n}=5$ ); and non-CPB controls $(\mathrm{n}=5)$. For the non-CPB protocol, after anesthesia for 6 hours, a thoracotomy was performed and myocardial tissue was harvested as described above.

All animals received humane care in compliance with the "Guide for the Care and Use of Laboratory Animals" prepared by the Association for Assessment and Accreditation of Laboratory Animal Care (March 1999), and approval was obtained from the Animal Resource Committee at the Hospital for Sick Children.

\section{Electron Microscopy}

The mitochondrial fraction was resuspended and fixed with $2 \%$ glutaraldehyde overnight. Mitochondria were postfixed, dehydrated, and embedded in Epon. Ultrathin sections were stained with lead citrate and uranyl acetate, and examined with a Hitachi H 7000 (Hitachi High-Technologies Corp, Schaumburg, Ill) transmission electron microscope at a magnification of $10,000 \times$.

\section{Mitochondrial Fractionation for Biochemical Analysis}

Mitochondrial fractionation was performed as described. ${ }^{6}$ Myocardium samples were mechanically homogenized and lysed. Unlysed cells and nuclei were removed by centrifugation at $750 \mathrm{~g}(5$ minutes $\times 2$ at $\left.4^{\circ} \mathrm{C}\right)$. The supernatant was spun at $8000 \mathrm{~g}(25$ minutes at $4^{\circ} \mathrm{C}$ ), and the resulting mitochondrial pellets were resuspended and frozen $\left(-80^{\circ} \mathrm{C}\right)$.

\section{Mitochondrial Bax Translocation}

Translocation of Bax from cytosol to mitochondria was examined by Western blot of mitochondrial protein fractions. Membranes were probed with anti-rabbit Bax (N-20, 1:1000; Santa Cruz Biotechnology, Inc, Santa Cruz, Calif). For protein loading control, the membranes were stripped and reprobed with anti-mouse mitochondrial porin (1:20,000; Molecular Probes, Inc, Carlsbad, Calif). Secondary antibodies were coupled to horseradish peroxidase (antirabbit immunoglobulin $[\operatorname{Ig}] \mathrm{G}$ 1:1000; anti-mouse $\operatorname{IgG} 1: 10,000$, Santa Cruz Biotechnology, Inc).

\section{Cytochrome c Release}

Mitochondrial outer membrane permeability was evaluated by measurement of cytochrome c release into the cytosol. After subcellular fractionation, cardiac cytosolic samples were standardized for protein concentration by a protein assay kit (Bio-Rad, Her- 
cules, Calif), denatured, and subsequently transferred to nitrocellulose membranes. The membrane was blocked with $5 \%$ nonfat milk and incubated overnight at $4^{\circ} \mathrm{C}$ with specific anti-mouse cytochrome c (1:2000; BD Biosciences Pharmingen, San Diego, Calif). For protein loading control, membranes were stripped and reprobed with anti-goat $\beta$-actin (1:5,000; Santa Cruz Biotechnology, Inc). Secondary antibodies were coupled to horseradish peroxidase (anti-mouse IgG 1:5,000; anti-goat IgG 1:10,000 Santa Cruz Biotechnology, Inc). Cytosolic cytochrome c was normalized to $\beta$-actin and cytosolic citrate synthase activity. ${ }^{3}$

\section{Fluorescent Immunohistochemistry and Confocal Imaging}

OCT-embedded transverse ventricular slices were cut into 5- $\mu \mathrm{m}$ serial sections fixed in acetone, and after blocking, mouse monoclonal anti-cytochrome c oxidase IV (COX IV) (1:250; BD Biosciences Clonetech, Palo Alto, Calif) was used. Sections were incubated with secondary antibody (Cy3-conjugated donkey antimouse IgG, 1:500; Jackson ImmunoResearch Laboratories, Inc, West Grove, Pa). Mouse monoclonal anti-cytochrome c (1:200; BD Biosciences Pharmingen) was used as the primary antibody for cytochrome c staining. Sections were immersed in secondary antibody (Cy2-conjugated donkey anti-mouse IgG, 1:200; Jackson ImmunoResearch Laboratories, Inc). The images from at least 3 different sections were acquired at $64 \times$ by a Zeiss LSM510 Multiphoton Laser Scanning Cofocal Microscope (Carl Zeiss, Jena, Germany), using the same pinhole setting, pixel format $(1024 \times$ 1024 , and scanning data depth $(0.8 \mu \mathrm{m})$. Double fluorescence for green and red channels was imaged using excitation of Argon$\mathrm{HeNel}$ at the wavelengths of 488 and $530 \mathrm{~nm}$. Fifty high-power fields from each animal were analyzed by imaging processing software (Velocity 3.0, Improvision, Lexington, Mass). The high intensity of red and green ( $>551$ voxels) was regarded as specific immunoreactive signals. The red (COX IV) was set as the reference signal, and the contribution of the green (cytochrome c) to the colocalization of the both signals was quantified as the overlap coefficient (Kx-green) and used to compare the diffusion of cytochrome c staining between groups. ${ }^{16}$

\section{Clark Electrode Oxygen Consumption Measurement}

Mitochondrial Complex I, II, and IV respiration were measured by the method of Ricci and colleagues ${ }^{17}$ using a Clark-type oxygen electrode (Instech Laboratories Inc, Plymouth Meeting, Pa). Oxygen consumption was measured in the presence of sequential administration of substrates and inhibitors (glutamate/malate for Complex I, rotenone/succinate for Complex II, antimycin/tetramethyl$p$-phenylenediamine/ascorbate for Complex IV) added in the following order and final concentration: $2.5 \mathrm{mmol} / \mathrm{L}$ glutamate, $2.5 \mathrm{mmol} / \mathrm{L}$ malate, $2 \mathrm{mmol} / \mathrm{L}$ adenosine diphosphate, $2 \mu \mathrm{mol} / \mathrm{L}$ rotenone, $5 \mathrm{mmol} / \mathrm{L}$ succinate, $1 \mu \mathrm{mol} / \mathrm{L}$ antimycin $\mathrm{A}$, and $1 \mathrm{mmol} / \mathrm{L}$ ascorbate with $0.4 \mathrm{mmol} / \mathrm{L} N, N, N^{\prime} N^{\prime}$-tetramethyl-p-phenylenediamine. Respiration rates are expressed as micromoles of molecular oxygen per milligram of mitochondrial protein.

\section{Deoxyuride-5' -Triphosphate Biotin Nick End Labeling Staining}

OCT-embedded transverse ventricular slices were cut into $10-\mu \mathrm{m}$ serial sections that were used for deoxyuride- 5 ' -triphosphate biotin nick end labeling (TUNEL). TUNEL was performed with the In Situ Cell Death Detection Kit (Roche Diagnostics, Indianapolis, Ind) according to the manufacturer's instructions. After TUNEL, all nuclei were counterstained with DAPI. The number of TUNELpositive myocytes and the number of total myocytes were counted in 25 random high-power fields $(\times 600)$ by a Leica (Deerfield, Ill) fluorescent microscope with OpenLab software (Improvision). Approximately 5000 myocytes were examined at least 3 different sections from each animal.

\section{Mitochondrial Calcium Tolerance: MPTP Opening Threshold Experiments}

Mitochondrial calcium tolerance experiments were determined by $\mathrm{Ca}^{2+}$-induced swelling of isolated cardiac mitochondria. ${ }^{18}$ Administration of exogenous calcium results in mitochondrial swelling, which is measured spectrophotometrically as a reduction in absorbance at $520 \mathrm{~nm}\left(\mathrm{~A}_{520}\right)$. Isolated cardiac mitochondria were resuspended in a swelling buffer to a final protein concentration of $0.22 \mathrm{mg} / \mathrm{mL}$. Two picomoles of $\mathrm{CaCl}_{2}$ were added every minute, and the percentage of changes of absorbance at $520 \mathrm{~nm}$ was recorded. After sufficient $\mathrm{Ca}^{2+}$ loading, an abrupt decrease in $\mathrm{A}_{520}$ absorbance seems to be the result of MPTP opening. The amount of $\mathrm{Ca}^{2+}$ needed to trigger this change is an indicator of the susceptibility of MPTP to $\mathrm{Ca}^{2+}$ overload. ${ }^{19}$

\section{Statistics}

Data are expressed as mean \pm standard error of the mean, and group comparisons were made with Fisher's least significant difference analysis of variance. A Tukey test was used for multiple post hoc comparisons.

\section{Results}

\section{Hemodynamic Analysis}

Hemodynamics (blood pressure, heart rate, central venous pressure), blood gas, hemoglobin, and venous oxygen saturations were monitored. At baseline, there were no differences between groups. During reperfusion, heart rate in the CCP group was increased at 1, 3, and 6 hours of reperfusion compared with the non-CPB group. Pretreatment with CsA prevented the heart rate increase. Arterial $\mathrm{pH}$ and base excess in the CCP group at 1 hour of reperfusion was decreased compared with the non-CPB group $(7.30 \pm 0.04$ and $-3.9 \pm 1.3$ vs $7.42 \pm 0.02$ and $0.9 \pm 0.5$, respectively; $P<.05)$. There were no differences in the value of $\mathrm{pH}$ and base excess between the non-CPB group and CCP + CsA groups. Hemodynamic data are included in Table E1.

\section{Electron Microscopy}

Representative electron microscopic images are shown in Figure 1. In contrast with the normal appearance of the mitochondrial cristae in the non-CPB mitochondria, there is extensive disarrangement in cristal architecture with narrowing of the mitochondrial intermembrane space in the CCP mitochondria. Despite the pronounced alterations in cristal architecture, the outer mitochondrial membranes ap- 

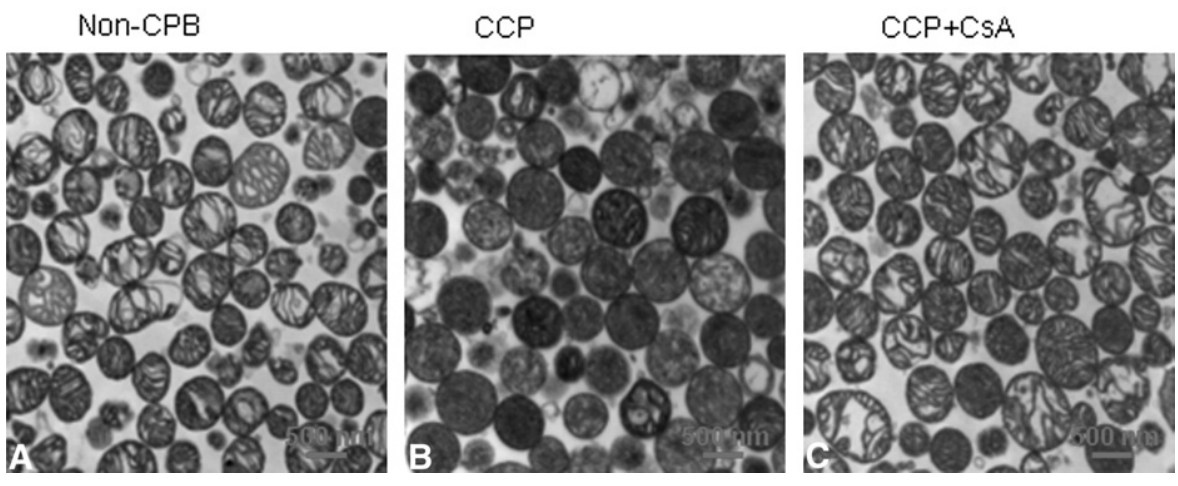

Figure 1. Representative electron micrographs of mitochondria isolated from the non-CPB hearts (A), CCP hearts $(B)$, and CCP + CsA hearts (C) $(\times 10,000)$. Compared with normal ultrastructure of mitochondria in nonCPB, distortion of cristae and narrowing of the intermembrane space were observed in the CCP mitochondria. Mitochondrial architecture was preserved in the CCP + CsA mitochondria. COX, Cytochrome c oxidase; CPB, cardiopulmonary bypass; $C C P$, cold crystalloid cardioplegia; $C s A$, cyclosporine $A$.

pear to be intact. In contrast, the mitochondrial cristal architecture was well preserved in the $\mathrm{CCP}+\mathrm{CsA}$ group.

\section{Mitochondrial Bax Translocation}

Mitochondrial Bax content was greater in CCP myocardium when compared with that in the non-CPB and CCP + CsA myocardium $(0.78 \pm 0.08 \mathrm{AU}$ vs $0.47 \pm 0.06 \mathrm{AU}$ and 0.57 $\pm 0.06 \mathrm{AU}, P<.01$ ), which is consistent with translocation of Bax from the cytosol to the mitochondria (Figure 2).

\section{Mitochondrial Permeabilization and}

\section{Cytochrome c Release}

Cytosolic cytochrome c concentrations were higher in $\mathrm{CCP}$ compared with non-CPB and $\mathrm{CCP}+\mathrm{CsA}$ myocardium $(10.49 \pm 0.56 \mathrm{AU}$ vs $7.16 \pm 0.61 \mathrm{AU}$ and $7.33 \pm 0.4 \mathrm{AU}$, respectively, $P<.05$; Figure 2$)$. Citrate synthase activity was not different between groups $\left(0.1 \pm 0.1 \mu \mathrm{mol}^{-1} / \mathrm{mg}^{-1}\right.$,
$0.1 \pm 0.2 \mu \mathrm{mol}^{-1} / \mathrm{mg}^{-1}$, and $0.09 \pm 0.1 \mu \mathrm{mol}^{-1} / \mathrm{mg}^{-1}$ protein for non- $\mathrm{CPB}, \mathrm{CCP}$, and $\mathrm{CCP}+\mathrm{CsA}$, respectively).

Fluorescent immunohistochemistry was used to compare the patterns of cytochrome c and COX IV staining (Figure 3, A). Release of cytochrome $\mathrm{c}$ from the mitochondria causes diffusion of the cytochrome c staining in the cytoplasm, and a stippled green pattern is produced. This pattern is prominent in the merged images from the $\mathrm{CCP}$ myocardium, whereas in non-CPB and CCP + CSA myocardium, only faint diffusion of cytochrome $\mathrm{c}$ staining in the merged images was found. The contribution of the green color intensity to the colocalization of these color voxels in the merged images was quantified by overlap coefficients $(\mathrm{Kx}$ green) (Figure 3. B). Compared with non-CPB and CCP + CsA myocardium, lower overlap coefficient ( $\mathrm{Kx}$-green) was found in CCP myocardium $(P<.01)$.

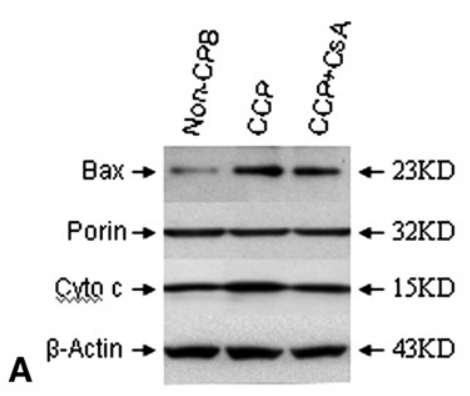

Bax translocation

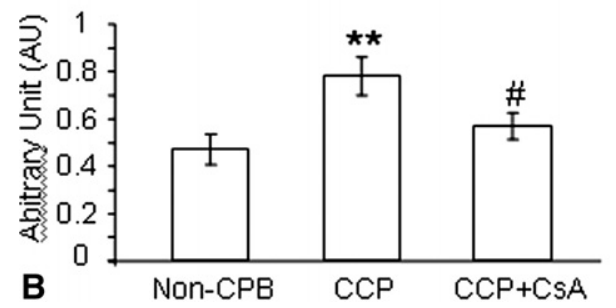

Cytochrome $c$ release

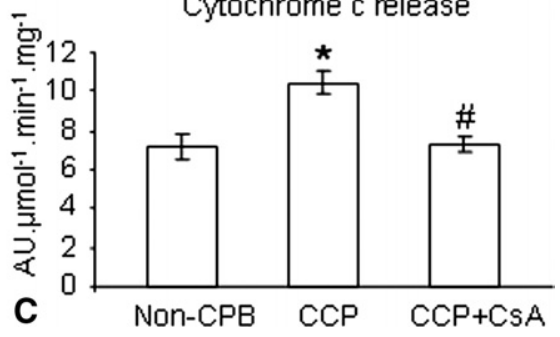

Figure 2. A, Representative Western blot of mitochondrial Bax and cytosolic cytochrome $c$ in non-CPB, ССР, and CCP + CsA hearts. B, Densitometric measurements of $B a x$ and cytochrome c in non-CPB, CCP, and CCP + CsA hearts. Increased concentrations of Bax in the mitochondrial fractions normalized by porin content are indicative of translocation of Bax to the mitochondria. C, Higher concentrations of cytochrome $\mathrm{c}$ in the cytosol standardized by $\beta$-actin and citrate synthase activity are indicative of mitochondrial permeabilization and release of cytochrome c. ${ }^{* *} P<.01$ and ${ }^{*} P<.05$ versus non-CPB and $\# P<.05$ versus CCP. COX, Cytochrome c oxidase; $C P B$, cardiopulmonary bypass; CCP, cold crystalIoid cardioplegia; $C s A$, cyclosporine $A$.

126 The Journal of Thoracic and Cardiovascular Surgery • January 2008 


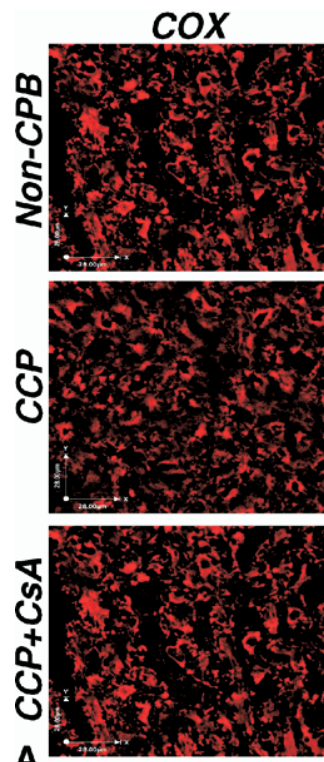
A
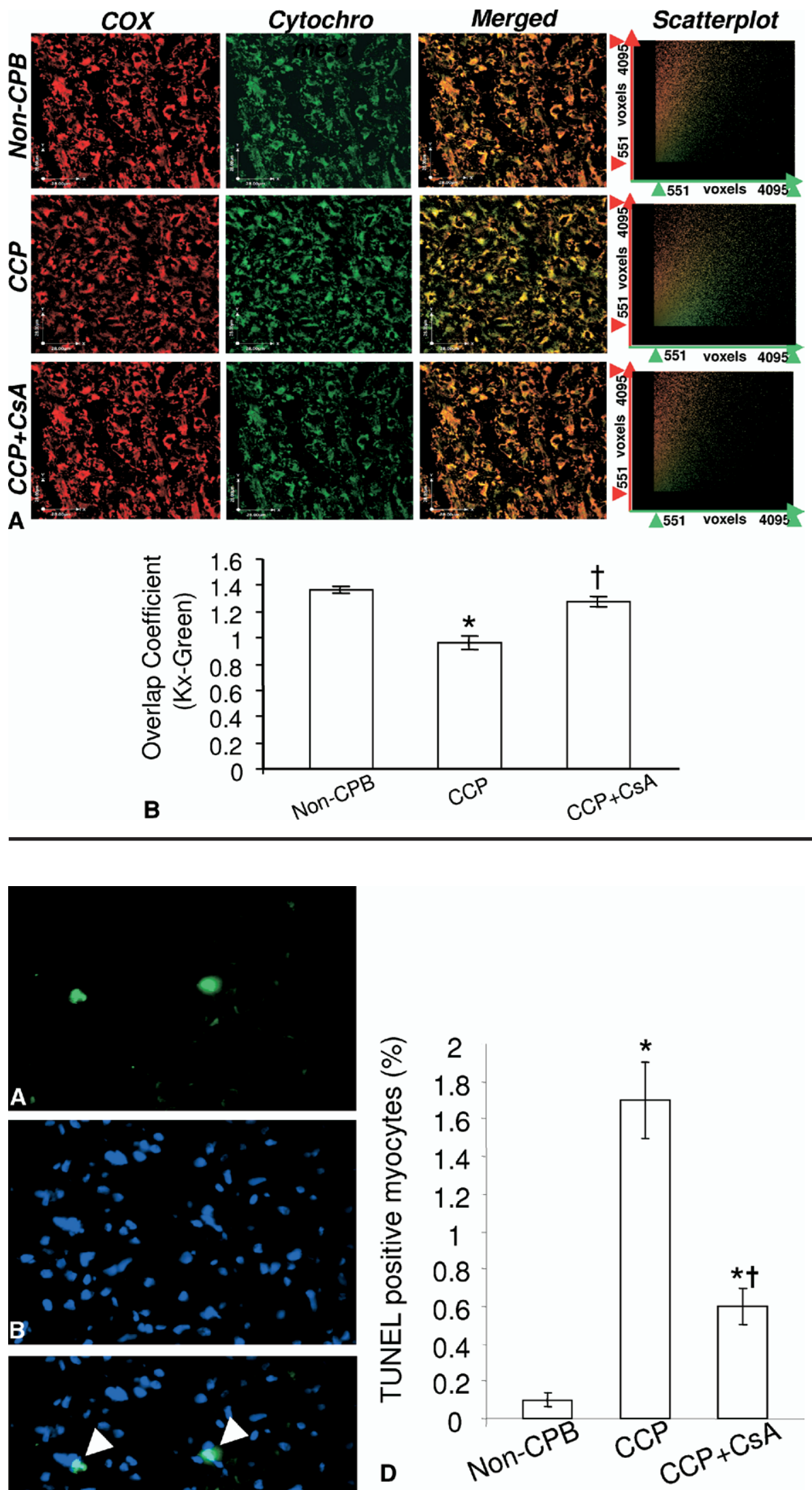
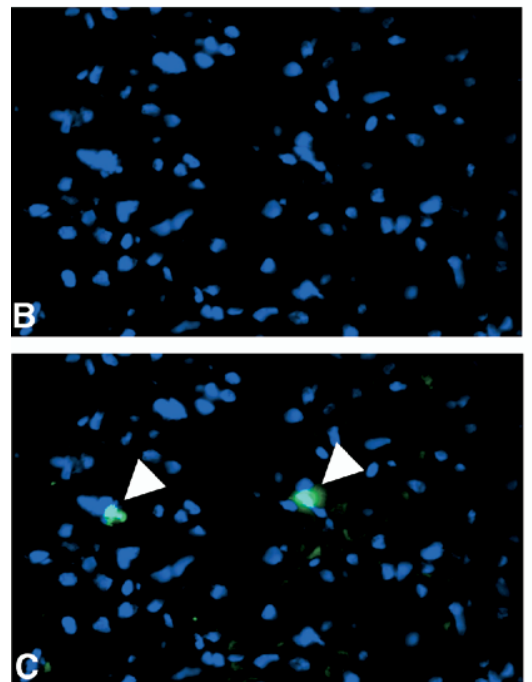

Figure 3. A, Immunofluorescence of COX IV and cytochrome $c$ in the myocardium from non-CPB, ССР, and ССР + CsA myocardium. In each panel, COX IV (mitochondria) is stained red, cytochrome $c$ is stained green, and the merged images are shown. Superimposition of red and green staining results in a brownish color that suggests retention of cytochrome $c$ in the non-CPB and CCP + CsA mitochondria. A fine diffuse green staining can be seen in the merged images of CCP myocardium, suggesting mitochondrial release of cytochrome c. Scatterplots display the intensity range of red and green pixels in the merged images of non-CPB, CCP, and CCP + CsA, as well as the various degrees of colocalization shown in orange and yellow. B, Quantification of the overlap coefficient (Kx-green) in the merged images for non-CPB, CCP, and CCP + CsA groups is shown. $\left({ }^{*} P<.01\right.$ vs non-CPB and $t P<.01$ vs CCP). COX, Cytochrome c oxidase; $C P B$, cardiopulmonary bypass; $C C P$, cold crystalloid cardioplegia; $C s A$, cyclosporine $A$.
Figure 4. A, Apoptotic nuclei are shown by TUNEL staining (green). B, Total nuclei were demarcated by DAPI staining (b/ue). C, Apoptotic nuclei in the merged image (arrows). D, Quantitative analysis of TUNEL staining $\left({ }^{*} P<.01\right.$ vs non-CPB and $t P<.05$ vs CCP). TUNEL, Deoxyuride-5' -triphosphate biotin nick end labeling; CPB, cardiopulmonary bypass; $C C P$, cold crystalloid cardioplegia; $C s A$, cyclosporine $A$. 
TABLE 1. Isolated mitochondrial oxygen consumption

\begin{tabular}{|c|c|c|c|c|c|c|c|c|c|}
\hline & \multicolumn{3}{|c|}{ Complex I } & \multicolumn{3}{|c|}{ Complex II } & \multicolumn{3}{|c|}{ Complex IV } \\
\hline & Non-CPB & ССР & CCP + CsA & Non-CPB & CCP & CCP + CsA & Non-CPB & ССР & $\mathrm{CCP}+\mathrm{CsA}$ \\
\hline State 2 & $0.33 \pm 0.02$ & $0.35 \pm 0.07$ & $0.31 \pm 0.01$ & $0.33 \pm 0.02$ & $0.35 \pm 0.07$ & $0.31 \pm 0.01$ & $0.33 \pm 0.02$ & $0.35 \pm 0.07$ & $0.31 \pm 0.01$ \\
\hline Ratio 3/2 & $22.40 \pm 2.01$ & $11.80 \pm 2.03^{*}$ & $25.20 \pm 1.91 \dagger$ & $7.60 \pm 0.40$ & $7.20 \pm 1.88$ & $7.40 \pm 1.17$ & $31.20 \pm 1.93$ & $25.20 \pm 6.26$ & $27.40 \pm 2.32$ \\
\hline
\end{tabular}

$\overline{C P B}$, Cardiopulmonary bypass; $C C P$, cold crystalloid cardioplegia; $C s A$, cyclosporine $\mathrm{A}$. ${ }^{*} P<.01$ versus non-CPB. $† P<.01$ versus $C C P$.

\section{Clark Electrode Oxygen Consumption Measurements}

Isolated mitochondrial oxygen consumption was assessed during state 2 respiration for mitochondrial Complexes I, II, and IV and was not different between groups, suggesting comparable levels of mitochondrial integrity after fractionation. Cardioplegic arrest was associated with diminished state 3 respiration at Complex I. The decrement in the ratio of state 3 to state 2 respiration was prevented by pretreatment with CsA $(22.4 \pm 2.0,11.8 \pm 2.0$, and $25.9 \pm 1.9$ for non-CPB, CCP, and CCP + CsA, respectively, $P<.01$ ) (Table 1). Complex II and Complex IV oxygen consumption rates were not different.

\section{TUNEL Staining}

TUNEL-positive nuclei were more frequent in CCP myocardium when compared with those in non-CPB and CsA groups $(1.7 \% \pm 0.2 \%$ vs $0.1 \% \pm 0.037 \%$ and $0.6 \% \pm$ $0.1 \%$, respectively; $P<.01$ and $P<.05$ ). There was no statistical difference in TUNEL-positive myocytes between the non-CPB and CCP + CsA groups (Figure 4).

\section{Mitochondrial Calcium Tolerance}

The amount of $\mathrm{Ca}^{2+}$ required to trigger MPTP opening of mitochondria was similar when comparing non-CPB and CCP mitochondria, suggesting that calcium overload is not a significant contributor to mitochondrial dysfunction 6 hours after cardioplegic arrest. CCP + CsA mitochondria were resistant to calcium-induced mitochondrial swelling, suggesting that CsA inhibited MPTP opening to a "supranormal" level (eg, beyond the resistance associated with non-CPB mitochondria). The calcium required to trigger MPTP opening was $136.6 \pm 4.74 \mu \mathrm{mol} / \mathrm{L} / \mathrm{mg}$ vs $84.8 \pm$ $6.48 \mu \mathrm{mol} / \mathrm{L} / \mathrm{mg}$ and $93.4 \pm 5.15 \mu \mathrm{mol} / \mathrm{L} / \mathrm{mg}$ protein for $\mathrm{CCP}+\mathrm{CsA}, \mathrm{CCP}$, and non-CPB, respectively $(P<.05)$.

\section{Discussion}

In the current study, postcardioplegic myocardium is associated with Bax translocation to the mitochondria, mitochondrial permeabilization, disarrangement of mitochondrial structure, release of cytochrome c into the cytoplasm, and a deficit of mitochondrial Complex I activity. Pretreatment with CsA, an MPTP opening inhibitor, prevents these changes, suggesting that prevention of MPTP opening may mitigate the mitochondrial effects of cardioplegic arrest.
The importance of these findings rests in the role of mitochondria as crucial cellular organelles controlling cell fate, ion homeostasis, and energy production. During the reperfusion period, mitochondrial structural and functional remodeling is associated with transmission of death signals (eg, cytochrome c) to apoptosis executioner proteins. ${ }^{5}$ The linkage between death stimuli and subsequent apoptosis is provided by multiple signaling pathways converging on the mitochondria, including Bcl-2 family proteins. ${ }^{20}$ Among the $\mathrm{Bcl}-2$ family of proteins, Bax is a known intermediate that can initiate permeabilization of mitochondria ${ }^{21}$ through direct pore-forming activity on the mitochondrial outer membrane and is associated with inhibition of mitochondrial respiratory chain activity. ${ }^{22,23}$ The translocation of Bax in the current study is consistent with a role for Bax in the mitochondrial release of cytochrome $\mathrm{c}$ after cardioplegic arrest.

In addition to triggering a postmitochondrial apoptotic cascade, cytochrome $\mathrm{c}$ is an important component of mitochondrial oxidative phosphorylation. A relative deficiency of mitochondrial cytochrome $\mathrm{c}$ has been reported to be responsible for deficits in mitochondrial respiratory chain activity and may thereby contribute to deficits in myocardial performance. ${ }^{24}$ Therefore, a rationale is provided for a myopreservation strategy focused on maintenance of mitochondrial structural and functional integrity. ${ }^{14,25}$ The MPTP is an important mediator of mitochondrial integrity, ${ }^{26}$ and inhibition of MPTP opening is associated with improved recovery of cardiac function after ischemic injury. ${ }^{27}$ Consequently, direct inhibition of MPTP opening with CsA may provide a valuable target for cardioprotection against ischemia/reperfusion injury.

In addition to MPTP inhibition, CsA also inhibits calcineurin, which may alter intracellular calcium homeostasis after ischemia/reperfusion injury. It is important to rule out indirect protection of the mitochondria through alterations in calcium handling. Calcineurin-mediated alterations in postreperfusion calcium loading are unlikely to be responsible for the findings of the present study for 2 reasons. First, Nathan and colleagues, ${ }^{15}$ using FK506 (a specific calcineurin inhibitor) in a similar in vitro cardioplegia model, demonstrated that the myopreservation effect of CsA is independent of calcineurin. In addition, in the 
present study, the calcium tolerance of mitochondrial MPTP opening was assessed using incremental administration of extramitochondrial calcium. The nearly identical tolerance of the CCP and non-CPB mitochondria suggests that postcardioplegia alterations in mitochondrial structure and function are not the result of calcineurin-mediated alterations in calcium loading of the mitochondria at 6 hours of reperfusion. The greater calcium tolerance of the CsA-treated mitochondria suggests that CsA inhibits MPTP opening after cardioplegic arrest. It is possible, however, that elevated cytosolic calcium concentrations earlier in the postoperative period (eg, <6 hours of reperfusion) may have played an important role in the observed alterations in mitochondrial structure and function.

The precise mechanism whereby cytochrome $\mathrm{c}$ is released across the outer mitochondrial membrane in the current study is not certain. MPTP opening has been described as occurring in 1 of 2 modes: transient and longlasting, the latter is often irreversible. ${ }^{9}$ Because the surface area of the inner mitochondrial membrane exceeds that of the outer membrane, extensive matrix swelling with longlasting MPTP opening can lead to the unfolding of cristae within the inner mitochondrial membrane, causing the outer membrane to rupture with irreversible release of cytochrome c. ${ }^{27}$ This mechanism does not appear to be present in the current study because the electron microscopy data demonstrated mitochondria in the CCP group that are highly remodeled in terms of cristal architecture but with little evidence of mitochondrial swelling or rupture of the outer mitochondrial membranes. In addition, citrate synthase activity was not increased in the CCP groups, suggesting that the structural integrity of the outer mitochondrial membrane was maintained in the CCP mitochondria. Therefore, if MPTP opening is the mechanism of cytochrome $\mathrm{c}$ release in the current study, a transient opening is more likely to be present than a long-lasting or permanent opening.

Another possible mechanism of cytochrome c release in the current study is direct Bax-mediated mitochondrial membrane permeabilization. ${ }^{28}$ Bax can directly interact with MPTP to regulate cytochrome $\mathrm{c}$ release $\mathrm{s}^{29,30}$ without any apparent loss of mitochondrial membrane potential. ${ }^{31} \mathrm{In}$ the current study, Bax translocation to the mitochondrial fraction was inhibited by CsA. Because transient MPTP opening has been demonstrated to contribute a feedforward signal to amplify Bax translocation to the mitochondria, ${ }^{32}$ the current data are consistent with CsA-mediated inhibition of this amplification step. Therefore, at least 2 possible mechanisms for cytochrome $\mathrm{c}$ release are consistent with the presented data: MPTP opening (and cytochrome c release) may be independent of Bax participation or MPTP opening may promote Bax translocation with subsequent Bax-mediated feedforward augmentation of mitochondrial permeabilization.

\section{Conclusions}

The current data suggest that MPTP-mediated alterations in mitochondrial structure and function are present after cardioplegic arrest and that inhibition of MPTP opening with CsA can ameliorate these changes. The "action" is focused at the level of the mitochondria, where postreperfusion cell survival, apoptosis, and necrosis signals are adjudicated, cell fate is determined, and energy for myocyte performance is generated. Better understanding of the cardinal role of mitochondria at the convergence point of these vital pathways will enhance myopreservation strategies for the cardiac surgeon.

\section{References}

1. Hoffman TM, Wernovsky G, Atz AM, Kulik TJ, Nelson DP, Chang $\mathrm{AC}$, et al. Efficacy and safety of milrinone in preventing low cardiac output syndrome in infants and children after corrective surgery for congenital heart disease. Circulation. 2003;107:996-1002.

2. Wernovsky G, Wypij D, Jonas RA, Mayer JE Jr, Hanley FL, Hickey $\mathrm{PR}$, et al. Postoperative course and hemodynamic profile after the arterial switch operation in neonates and infants. A comparison of low-flow cardiopulmonary bypass and circulatory arrest. Circulation. 1995;92:226-35.

3. Schmitt JP, Schroder J, Schunkert H, Birnbaum DE, Aebert H. Role of apoptosis in myocardial stunning after open heart surgery. Ann Thorac Surg. 2002;73:1229-35.

4. Hammel JM, Caldarone CA, Van Natta TL, Wang LX, Welke KF, Li $\mathrm{W}$, et al. Myocardial apoptosis after cardioplegic arrest in the neonatal lamb. J Thorac Cardiovasc Surg. 2003;125:1268-75.

5. Crow MT, Mani K, Nam YJ, Kitsis RN. The mitochondrial death pathway and cardiac myocyte apoptosis. Circ Res. 2004;95:957-70.

6. Caldarone CA, Barner EW, Wang L, Karimi M, Mascio CE, Hammel $\mathrm{JM}$, et al. Apoptosis-related mitochondrial dysfunction in the early postoperative neonatal lamb heart. Ann Thorac Surg. 2004;78:948-55.

7. Wang L, Kinnear C, Hammel JM, Zhu W, Hua Z, Mi W, Caldarone CA. Preservation of mitochondrial structure and function after cardioplegic arrest in the neonate using a selective mitochondrial KATP channel opener. Ann Thorac Surg. 2006;81:1817-23.

8. Griffiths EJ, Halestrap AP. Mitochondrial non-specific pores remain closed during cardiac ischaemia, but open upon reperfusion. Biochem J. 1995;307:93-8.

9. Lemasters JJ, Qian T, Elmore SP, Trost LC, Nishimura Y, Herman B, et al. The mitochondrial permeability transition in cell death: a common mechanism in necrosis, apoptosis and autophagy. Biochim Biophys Acta. 1998;1366:177-96.

10. Baines CP, Song CX, Zheng YT, Wang GW, Zhang J, Wang OL, et al. Protein kinase Cepsilon interacts with and inhibits the permeability transition pore in cardiac mitochondria. Circ Res. 2003;92:873-80.

11. Nakagawa T, Shimizu S, Watanabe T, Yamaguchi O, Otsu K, Yamagata $\mathrm{H}$, et al. Cyclophilin D-dependent mitochondrial permeability transition regulates some necrotic but not apoptotic cell death. Nature. 2005;434:652-8.

12. Akao M, O'Rourke B, Kusuoka H, Teshima Y, Jones SP, Marban E. Differential actions of cardioprotective agents on the mitochondrial death pathway. Circ Res. 2003;92:195-202.

13. Halestrap AP, Connern CP, Griffiths EJ, Kerr PM. Cyclosporin A binding to mitochondrial cyclophilin inhibits the permeability transition pore and protects hearts from ischaemia/reperfusion injury. Mol Cell Biochem. 1997;174:167-72.

14. Argaud L, Gateau-Roesch O, Muntean D, Chalabreysse L, Loufouat J, Robert D, et al. Specific inhibition of the mitochondrial permeability transition prevents lethal reperfusion injury. J Mol Cell Cardiol. 2005; 38:367-74. 
15. Nathan M, Friehs I, Choi YH, Cowan DB, Cao-Danh H, McGowan FX, et al. Cyclosporin A but not FK-506 protects against dopamineinduced apoptosis in the stunned heart. Ann Thorac Surg. 2005;79: 1620-6.

16. Zinchuk V, Zinchuk O, Okada T. Experimental LPS-induced cholestasis alters subcellular distribution and affects colocalization of Mrp2 and Bsep proteins: a quantitative colocalization study. Microsc Res Tech. 2005;67:65-70.

17. Ricci JE, Gottlieb RA, Green DR. Caspase-mediated loss of mitochondrial function and generation of reactive oxygen species during apoptosis. J Cell Biol. 2003;160:65-75.

18. Wang G, Liem DA, Vondriska TM, Honda HM, Korge P, Pantaleon DM, et al. Nitric oxide donors protect murine myocardium against infarction via modulation of mitochondrial permeability transition. Am J Physiol Heart Circ Physiol. 2005;288:H1290-5.

19. Argaud L, Gateau-Roesch O, Raisky O, Loufouat J, Robert D, Ovize M. Postconditioning inhibits mitochondrial permeability transition. Circulation. 2005;111:194-7.

20. Danial NN, Korsmeyer SJ. Cell death: critical control points. Cell. 2004;116:205-19.

21. Puthalakath H, Strasser A. Keeping killers on a tight leash: transcriptional and post-translational control of the pro-apoptotic activity of BH3-only proteins. Cell Death Differ. 2002;9:505-12.

22. Wei MC, Zong WX, Cheng EH, Lindsten T, Panoutsakopoulou V, Ross AJ, et al. Proapoptotic BAX and BAK: a requisite gateway to mitochondrial dysfunction and death. Science. 2001;292:727-30.

23. Appaix F, Guerrero K, Rampal D, Izikki M, Kaambre T, Sikk P, et al. Bax and heart mitochondria: uncoupling and inhibition of respiration without permeability transition. Biochim Biophys Acta. 2002;1556: 155-67.

24. Mootha VK, Wei MC, Buttle KF, Scorrano L, Panoutsakopoulou V, Mannella CA, et al. A reversible component of mitochondrial respi- ratory dysfunction in apoptosis can be rescued by exogenous cytochrome c. EMBO J. 2001;20:661-71.

25. Rousou AJ, Ericsson M, Federman M, Levitsky S, McCully JD. Opening of mitochondrial KATP channels enhances cardioprotection through the modulation of mitochondrial matrix volume, calcium accumulation, and respiration. Am J Physiol Heart Circ Physiol. 2004;287:H1967-76

26. Sharov VG, Todor A, Khanal S, Imai M, Sabbah HN. Cyclosporine A attenuates mitochondrial permeability transition and improves mitochondrial respiratory function in cardiomyocytes isolated from dogs with heart failure. J Mol Cell Cardiol. 2007;42:150-8.

27. Weiss JN, Korge P, Honda HM, Ping P. Role of the mitochondrial permeability transition in myocardial disease. Circ Res. 2003;93: 292-301.

28. Antonsson B, Montessuit S, Lauper S, Eskes R, Martinou JC. Bax oligomerization is required for channel-forming activity in liposomes and to trigger cytochrome c release from mitochondria. Biochem J. 2000;345:271-8.

29. Shimizu S, Narita M, Tsujimoto Y. Bcl-2 family proteins regulate the release of apoptogenic cytochrome $\mathrm{c}$ by the mitochondrial channel VDAC. Nature. 1999;399:483-7.

30. Narita M, Shimizu S, Ito T, Chittenden T, Lutz RJ, Matsuda H, et al. Bax interacts with the permeability transition pore to induce permeability transition and cytochrome c release in isolated mitochondria. Proc Natl Acad Sci U S A. 1998;95:14681-6.

31. Bossy-Wetzel E, Newmeyer DD, Green DR. Mitochondrial cytochrome $\mathrm{c}$ release in apoptosis occurs upstream of DEVD-specific caspase activation and independently of mitochondrial transmembrane depolarization. EMBO J. 1998;17:37-49.

32. De Giorgi F, Lartigue L, Bauer MK, Schubert A, Grimm S, Hanson GT, et al. The permeability transition pore signals apoptosis by directing Bax translocation and multimerization. FASEB J. 2002;16:607-9.

\section{JTCVS On-Line Manuscript Submission and Review}

The Journal of Thoracic and Cardiovascular Surgery requires authors and reviewers to submit all new and revised manuscripts and reviews via Editorial Manager. Point your browser to http://jtcvs.editorialmanager.com, log in as author or reviewer (as appropriate), and follow the instructions provided.

To retrieve your username and password, click "Forget your password?" on the Editorial Manager log-in page.

If you have questions or experience problems uploading your manuscript or review, please contact the editorial office:

Telephone: 978-299-4516

E-mail: rwalther@prri.com 
Table E1. Hemodynamics and blood gas in piglet model of cardioplegic arrest

\begin{tabular}{|c|c|c|c|c|c|c|}
\hline & \multicolumn{3}{|c|}{ Baseline } & \multicolumn{3}{|c|}{ 1-h Reperfusion } \\
\hline & Sham & ССР & CsA & Sham & СCP & CsA \\
\hline HR (beats/min) & $115 \pm 10.9$ & $122 \pm 6.8$ & $124 \pm 6.1$ & $115 \pm 10.5$ & $151 \pm 12.8^{*}$ & $112 \pm 6.1 \dagger$ \\
\hline $\mathrm{SBP}(\mathrm{mm} \mathrm{Hg})$ & $65 \pm 3.6$ & $60 \pm 4.0$ & $66 \pm 1.9$ & $59 \pm 4.5$ & $57 \pm 1.2$ & $59 \pm 4.8$ \\
\hline $\mathrm{DBP}(\mathrm{mm} \mathrm{Hg})$ & $40 \pm 1.6$ & $36 \pm 3.9$ & $42 \pm 0.9$ & $37 \pm 3.1$ & $31 \pm 2.4$ & $31 \pm 2.8 \ddagger$ \\
\hline CVP $(\mathrm{mm} \mathrm{Hg})$ & $7.2 \pm 0.6$ & $5.2 \pm 0.8$ & $6.2 \pm 0.7$ & $7.2 \pm 0.5$ & $6.6 \pm 0.7$ & $6.6 \pm 1.1$ \\
\hline Body temperature $\left({ }^{\circ} \mathrm{C}\right)$ & $37 \pm 0.0$ & $38 \pm 0.5$ & $38 \pm 0.3$ & $37 \pm 0.1$ & $37 \pm 0.8$ & $37 \pm 0.3$ \\
\hline $\mathrm{K}(\mathrm{mmol} / \mathrm{L})$ & $4.0 \pm 0.2$ & $3.9 \pm 0.1$ & $3.6 \pm 0.2$ & $4.7 \pm 0.3$ & $3.7 \pm 0.2^{*}$ & $3.6 \pm 0.2^{*}$ \\
\hline $\mathrm{Ca}(\mathrm{mmol} / \mathrm{L})$ & $1.4 \pm 0.0$ & $1.2 \pm 0.1$ & $1.3 \pm 0.1$ & $1.5 \pm 0.0$ & $1.4 \pm 0.1$ & $1.3 \pm 0.0^{*}$ \\
\hline $\mathrm{tHb}(\mathrm{g} / \mathrm{dL})$ & $9.4 \pm 0.3$ & $10.1 \pm 0.4$ & $9.3 \pm 0.7$ & $9.7 \pm 0.6$ & $10.5 \pm 0.8$ & $12.0 \pm 1.0$ \\
\hline $\mathrm{pH}$ & $7.49 \pm 0.02$ & $7.49 \pm 0.04$ & $7.50 \pm 0.04$ & $7.42 \pm 0.02 \ddagger$ & $7.30 \pm 0.04^{*} \ddagger$ & $7.36 \pm 0.02 \ddagger$ \\
\hline $\mathrm{Po}_{2}(\mathrm{~mm} \mathrm{Hg})$ & $516 \pm 9.7$ & $493 \pm 20$ & $503 \pm 26$ & $435 \pm 53.6$ & $272 \pm 70 \ddagger$ & $281 \pm 88 \ddagger$ \\
\hline $\mathrm{PcO}_{2}(\mathrm{~mm} \mathrm{Hg})$ & $34 \pm 2.3$ & $34 \pm 3.4$ & $29 \pm 4.1$ & $39 \pm 1.6$ & $47 \pm 3.6 \ddagger$ & $42 \pm 3.3$ \\
\hline $\mathrm{HCO}_{3}(\mathrm{mmol} / \mathrm{L})$ & $26 \pm 0.5$ & $25 \pm 1.3$ & $24 \pm 1.7$ & $25 \pm 0.6$ & $22 \pm 0.8^{*} \ddagger$ & $23 \pm 1.2$ \\
\hline $\mathrm{BE}(\mathrm{mmol} / \mathrm{L})$ & $3.1 \pm 0.3$ & $2.2 \pm 1.2$ & $1.8 \pm 1.3$ & $0.9 \pm 0.5 \ddagger$ & $-3.9 \pm 1.3^{*} \ddagger$ & $-1.6 \pm 0.9$ \\
\hline $\mathrm{SAO}_{2}(\%)$ & $100 \pm 0.0$ & $100 \pm 0.0$ & $100 \pm 0.4$ & $100 \pm 0.0$ & $98 \pm 1.8$ & $100 \pm 0.0$ \\
\hline $\mathrm{SvO}_{2}(\%)$ & $86 \pm 1.0$ & $82 \pm 2.5$ & $83 \pm 4.0$ & $68 \pm 2.1 \ddagger$ & $55 \pm 9.7 \ddagger$ & $60 \pm 6.4 \ddagger$ \\
\hline
\end{tabular}

$H R$, Heart rate; $S B P$, systolic blood pressure; $D B P$, diastolic blood pressure; $C V P$, central venous blood pressure; $t H b$, total hemoglobin; $B E$, base excess; $\mathrm{SAO}_{2}$, arterial oxygen saturation; $\mathrm{SvO}_{2}$, venous oxygen saturation in non-CPB, CCP, and CCP + CsA-treated piglets. ${ }^{*} P<.05$ versus non-CPB at the same time point. $† P<.05$ versus CCP at same time point. $¥ P<.05$ versus non-CPB at baseline. 
TABLE E1. Continued

\begin{tabular}{|c|c|c|c|c|c|}
\hline \multicolumn{3}{|c|}{ 3-h Reperfusion } & \multicolumn{3}{|c|}{ 6-h Reperfusion } \\
\hline Sham & CCP & CsA & Sham & CCP & CsA \\
\hline $117 \pm 9.3$ & $130 \pm 14.5^{*}$ & $112 \pm 10.9 \dagger$ & $125 \pm 8.0$ & $152 \pm 9.8^{*}$ & $115 \pm 11.4 \dagger$ \\
\hline $31 \pm 2.3 \ddagger$ & $28 \pm 3.4$ & $32 \pm 2.8 \ddagger$ & $26 \pm 1.8 \ddagger$ & $25 \pm 2.3$ & $30 \pm 5.2 \ddagger$ \\
\hline $7.8 \pm 0.4$ & $6.8 \pm 0.7$ & $6.8 \pm 1.2$ & $8.0 \pm 0.4$ & $6.6 \pm 0.8$ & $7.0 \pm 1.2$ \\
\hline $37 \pm 0.2$ & $37 \pm 0.7$ & $37 \pm 0.4$ & $37 \pm 0.2$ & $37 \pm 0.6$ & $37 \pm 0.5$ \\
\hline $1.3 \pm 0.0$ & $1.4 \pm 0.1$ & $1.4 \pm 0.1$ & $1.4 \pm 0.1$ & $1.4 \pm 0.1$ & $1.3 \pm 0.0$ \\
\hline $9.3 \pm 0.8$ & $10.9 \pm 1.2$ & $13.5 \pm 1.1^{*} \ddagger$ & $9.0 \pm 0.7$ & $10.9 \pm 1.2$ & $14.3 \pm 1.5^{*} \ddagger$ \\
\hline $7.44 \pm 0.02 \ddagger$ & $7.41 \pm 0.03$ & $7.40 \pm 0.01 \ddagger$ & $7.42 \pm 0.02 \ddagger$ & $7.43 \pm 0.03$ & $7.42 \pm 0.02 \ddagger$ \\
\hline $474 \pm 9.7$ & $340 \pm 73.9$ & $289 \pm 76.3 \ddagger$ & $474 \pm 18.5$ & $349.4 \pm 78.9$ & $444 \pm 33.5$ \\
\hline $34 \pm 1.6$ & $41 \pm 3.9$ & $50 \pm 11.2 \ddagger$ & $39 \pm 2.2$ & $40 \pm 3.4$ & $35 \pm 1.3$ \\
\hline $23 \pm 0.6 \ddagger$ & $25 \pm 0.9$ & $23 \pm 1.6$ & $25 \pm 0.7$ & $26 \pm 0.5$ & $23 \pm 1.5$ \\
\hline
\end{tabular}

